DOI: $10.17234 / S R A Z .65 .53$

UDK: 81'282.2(497.5 Istra)

UDK: 811.131.1'282.2(497.5 Istra)

UDK: 811.163.42'282.2(497.5 Istra)

UDK: 811.163.6'282.2(497.5 Istra)

Preliminary communication

Ricevuto il 27 aprile 2020

Approvato per la pubblicazione il 25 novembre 2020

\title{
Dialetti in contatto nella Regione Istriana. Metodi d'indagine per un Archivio della memoria linguistica e culturale dell'Istria
}

\author{
Eliana Moscarda Mirković \\ Nada Poropat Jeletić \\ Università degli Studi 'Juraj Dobrila' di Pola \\ eliana.moscarda.mirkovic@unipu.hr \\ nada.poropat.jeletic@unipu.hr
}

Il contributo presenta sinteticamente le fasi della ricerca finora condotta nell'ambito del progetto Archivio della memoria linguistica e culturale dell'Istria, realizzato in collaborazione tra l'Università degli Studi 'Juraj Dobrila' di Pola e l'Università degli Studi di Trieste. Il progetto prevede la creazione di un archivio linguistico e culturale, attraverso la raccolta di fonti per formare una prima base di conoscenze e informazioni, selezionate attraverso una campagna di colloqui e interviste, con i depositari del sapere linguistico e culturale istriano.

Parole chiave: archivio, Istria, lingue minoritarie, lingue in contatto, dialetti

\section{Introduzione}

Il patrimonio culturale materiale e immateriale della Regione Istriana necessita di un lavoro di riordino, di riscoperta e di valorizzazione e rappresenta una risorsa indispensabile per il bene comune delle genti istriane nonché per la sua fruizione e promozione. Occorre intervenire su questi 'beni' nell'immediato presente, attraverso una seria programmazione, in quanto col passare del tempo riesce sempre più arduo recuperare le parlate, le tradizioni e le culture dei nostri antenati, che difficilmente si possono tramandare senza le testimonianze di chi le ha vissute. Nell'ideazione del progetto Archivio della memoria linguistica e culturale dell'Istria (AMLCI) sono state coniugate queste riflessioni a una necessità di cui si parla sempre più spesso, ovvero quella di salvaguardare e valorizzare le lingue minoritarie (tema già da tempo affrontato a livello comunitario europeo e ultimamente anche da parte della stampa e di importanti esponenti del mondo culturale e politico regionale).

Il progetto AMLCI è stato avviato nel 2018 grazie alla collaborazione tra l’Università degli Studi 'Juraj Dobrila' di Pola e l’Università degli Studi di Trieste 
ed è finanziato con fondi speciali dalla Regione Friuli Venezia Giulia per il tramite dell'Università Popolare di Trieste ${ }^{1}$. Il progetto prevede la raccolta di fonti per formare una prima base di conoscenze e informazioni, selezionate attraverso una campagna di colloqui e interviste con i depositari del sapere linguistico e culturale istriano. L'impostazione è volta a difendere e a valorizzare le ricchezze linguistiche dialettali di cui il territorio istriano dispone. È evidente, infatti, che la globalizzazione e il mutare delle condizioni economiche e sociali rischiano di far scomparire per sempre un patrimonio immenso di pratiche e di saperi, che sono solo apparentemente umili e che di fatto sono l'ossatura portante di qualsiasi approfondimento storico.

Quelle illustrate in questa sede sono informazioni relative alla conduzione delle prime fasi di ricerca di quello che sarà un progetto su vasta scala e di durata pluriennale, condotto sul modello dell'Atlante Storico-Linguistico-Etnografico Friulano diretto da Gian Battista Pellegrini (1972 ssg.) e del Slovenski dialektološki leksikalni atlas slovenske Istre (2 vol.) diretto da Franco Crevatin (Cossutta e Crevatin: 2006). Il gruppo di lavoro che opera in seno al progetto ha riconosciuto l'opportunità di interventi di ricerca sistematici sui dialetti dell'Istria ${ }^{2}$, regione che resta ancor oggi da esplorare per quanto riguarda la sua variazione dialettale e sociolinguistica, per ricostruirne la memoria linguistica e culturale nonché costituire gradualmente un archivio accessibile sia agli studiosi sia agli interessati; un archivio che possa documentare identità e storia delle genti istriane. Il progetto si è posto come obiettivo quello di studiare, attraverso le parole locali, i diversi aspetti della vita e del lavoro tradizionali delle comunità dell'Istria (soprattutto quelli relativi alla casa e all'agricoltura), in buona parte ormai obsoleti. Si prevede che i dati raccolti forniranno il riflesso delle profonde trasformazioni sociali, culturali e linguistiche che hanno interessato questo territorio.

In questa sede, per motivi di spazio redazionale, non ci è possibile ripercorrere in modo esauriente la struttura del progetto, bensì cercheremo di focalizzare l'attenzione, come si è detto, sulle prime tappe della ricerca condotte fino a questo momento.

1 Parte della ricerca è supportata finanziariamente pure dalla Fondazione Croata per la Scienza nell'ambito del progetto scientifico Višerazinski pristup govornom diskursu u jezičnom razvoju (L'approccio multilivello all'analisi del discorso nello sviluppo del linguaggio UIP-2017-05-6603).

2 Importanti studi sui dialetti italiani dell'Istria sono stati condotti da: Balbi e Moscarda Budić (2003); Buršić-Giudici (2009, 2011); Buršić-Giudici e Orbanich (2009); Cergna (2015); Cernecca (1967, 1976, 1986); Cortelazzo (1972); Crevatin (1982, 1999); Dalla Zonca (1978); Deanović (1955, 1960, 1962); Dussich (2019); Filipi (1989b, 1993, 1994, 1996); Filipi e Buršić-Giudici (1998, 2012); Glavinić (2000); Manzini e Rocchi (1995); Pellizzer e Pellizzer (1992); Prati (1951); Rosamani (21999); Tekavčić (1976); Todorović $(2019,2020)$. 


\section{Mappatura e raccolta dei dati}

Allo scopo di ottenere un quadro completo ed esaustivo della realtà su cui si vuole intervenire, nella prima fase della ricerca è stata realizzata la mappatura dei punti d'indagine in cui effettuare le interviste. Il progetto prevede la messa a punto di un archivio di interviste e lemmi dialettali raccolti nelle seguenti località: Buie, Cittanova, Dignano, Fasana, Gallesano, Glavice, Grisignana, Lanišće, Pedena, Pinguente, Pirano, Portole, Rovigno, Santa Croce di Aidussina, Scuiari, Sissano, Umago, Valle e Verteneglio.

Nella seconda fase si è creato uno strumento di raccolta dei dati comprendente le voci legate al campo semantico della 'casa'. L'esito finale ha prodotto uno strumento (griglia) composto da 280 lemmi in lingua italiana, con altrettante domande di supporto in dialetto istroveneto e in lingua croata volte ad agevolare la conduzione di interviste semi-strutturate. Lo strumento è stato creato sulla base dell'Atlante Linguistico Italiano (Bartoli et al.: 1995 ssg.), dell' Atlante linguistico istrioto (Filipi e Buršić-Giudici: 1998) e dell' Atlante Linguistico Istroveneto (Filipi e Buršić-Giudici: 2012), con aggiunte di lemmi ed espressioni alla lista base della griglia da parte dei coordinatori e collaboratori, tenendo conto della specificità territoriale dell'indagine.

La terza fase del progetto ha finora riguardato la ricerca sul campo in otto delle diciannove località previste. Le tecniche di intervista più comunemente impiegate nella ricerca dialettologica sono sostanzialmente tre: l'intervista direttiva, la non-direttiva e quella interattiva (cfr. Matranga 2002). Come metodi di ricerca per l'AMLCI si stanno usando interviste collettive non strutturate a risposta libera e quando necessario interviste semi-strutturate a risposta libera, privilegiando il parlato spontaneo degli intervistati. A tal fine gli intervistatori sono stati istruiti, con la messa a punto di un protocollo di inchiesta ${ }^{4}$, a non considerare la griglia/il questionario un punto fermo o "un porto tranquillo nel quale rifugiarsi o una difesa di cui farsi scudo" (D'Agostino/Paternostro 2006: 30), ma un supporto da utilizzare nelle fasi più problematiche dell'inchiesta, il punto di partenza di un percorso da compiere insieme agli intervistati per giungere a definizioni attendibili laddove l'intervistato non fosse in grado di riprodurre la voce dialettale per il corrispettivo italiano/croato. La tipologia delle interviste punta a salvaguardare la spontaneità e l'autenticità delle risposte dell'informatore, limitando fortemente la possibilità dell'intervistatore di intervenire nel merito delle risposte. Sin da subito le interviste hanno presentato una loro complessità interna, in quanto sia gli informatori (ma ovviamente anche i raccoglitori) sono stati costretti a muoversi, all'interno di situazioni linguistiche diversificate, fra dialetti istroveneti, istrioti, ciacavi e caicavi. Tale

3 Buie, Gallesano, Grisignana, Pinguente, Rovigno, Sissano, Umago, Valle.

4 Il protocollo d'inchiesta specifica il modo in cui vanno somministrate le domande, suggerisce alcune strategie da adottare per l'elicitazione dei dati linguistici e autorizza gli intervistatori ad allontanarsi, se necessario, dalle domande della griglia nei casi in cui la loro formulazione ostacoli il parlato spontaneo. 
complessità sta però consentendo di raccogliere materiale utilizzabile anche in maniera comparativa.

Quello che fino a questo momento è emerso dalle interviste condotte, è una generale volontà degli informatori di ampliare le risposte alle domande, per dar vita a contesti di vita vissuta, con livelli di spontaneità ben superiori a quelli che si potrebbero ottenere attraverso interviste direttive. Ciò ha indotto gli intervistatori ad apportare cambiamenti nella formulazione di alcune domande e ad aggiungere quesiti e lemmi nuovi a quelli già esistenti nella griglia.

Le ricerche negli ultimi anni hanno ampliato la gamma delle variabili individuali che possono essere legate al comportamento linguistico. Oltre a 'sesso', 'istruzione', 'cultura familiare' si è usato un indicatore complesso quale 'l'ambizione individuale', oppure ancora l'appartenenza a una tipologia di 'rete sociale', o ancora variabili di tipo psicologico (cfr. Berruto 2010). All'interno di quello che può essere considerato un continuo raffinamento delle capacità di cogliere quella variabilità linguistica che, come efficacemente diceva André Martinet "comincia dalla porta accanto", l'età continua a confermarsi come fattore essenziale negli studi variazionisti (Martinet 2008: CVII).

Il profilo della ricerca dell'AMLCI è geograficamente ristretto, in quanto si concentra solo sulle testimonianze linguistiche fornite dalle persone nate e/o residenti sul territorio delle località sopra menzionate. Pertanto si è privilegiata, nelle nostre considerazioni, la variabile 'età' ${ }^{5}$ siccome gli informatori hanno vissuto i momenti linguisticamente fondamentali della loro esistenza confrontandosi con varianti linguistiche diverse e spesso interferenti. Per alcuni aspetti le loro esperienze possono restituire la complessa e non sempre lineare stratificazione nella Regione Istriana di momenti storici diversi.

Il numero degli intervistati è al momento di diciotto persone, di cui nove uomini e nove donne. La data di nascita degli informatori scelti si distribuisce tra gli anni '30 e '60 dello scorso secolo. Si tratta, quindi, di una categoria di persone, quasi sempre di età avanzata, che hanno valorizzato la forma della griglia attraverso ricordi, commenti, digressioni e precisazioni. Le interviste si sono svolte prevalentemente nelle abitazioni dei singoli intervistati o presso le sedi delle Comunità degli Italiani in loco, che hanno fatto da tramite nel contattare gli intervistati. Si è limitato a un minimo di due e a un massimo di quattro il numero degli intervistati per agevolare gli interventi correttivi e il recupero memoriale dei dati. Si è ottenuto così, in base alle caratteristiche desiderate e desiderabili per riempire la griglia, dei dati confermati da persone di caratteristiche simili o identiche, ma la cui testimonianza, vista da un'altra prospettiva, ha rappresentato un valido contributo alla conferma dei dati oggetto d'analisi.

Gli informatori sono stati intervistati in esclusiva presenza del raccoglitore che ha avuto come primo compito quello di mettere a proprio agio l'interlocutore, per coinvolgerlo in un dialogo il più naturale possibile e fargli così dimenticare la

5 La correlazione della variabile 'sesso' con i dati attesi della ricerca dell' AMLCI è marginale. Il linguaggio femminile non si diversifica da quello maschile nella fascia d'età presa in esame. 
presenza dell'intervistatore. In base alle indicazioni di Gobo, agli intervistati sono state date istruzioni relative a come recuperare le informazioni in memoria, come costruire un giudizio in conformità alle aspettative dei ricercatori, come rispondere in modo appropriato, come percepire la richiesta di informazioni (Gobo ${ }^{32002: 46) .}$ Il risultato ottenuto è un pieno coinvolgimento, anche emotivo, dell'intervistato nel corso dell'intervista, soprattutto nel contesto delle domande relative al lessico arcaico dove le singole voci sono state contestualizzate all'interno di un quadro di esperienze, soprattutto passate, in cui il dialetto e i singoli lemmi sono diventati espressioni tangibili di vita reale. Trovandosi di fronte allo strumento della griglia, gli intervistati ne hanno ampliato sovente i contorni. Si è arrivati, pertanto, a cambiamenti nella formulazione di alcune domande o all'aggiunta di alcune parole ed espressioni. Le domande del questionario, messe le une accanto alle altre, stanno offrendo un sorprendente mosaico di conoscenze e aspetti che fornisce precise direzioni d'indagine nel repertorio linguistico istriano.

Riportiamo qui di seguito due esempi, uno relativo a un lemma e l'altro a un modo di dire, aggiunti nella griglia in seguito alle interviste condotte:

mùla s. f. - 'specie di sanguinaccio'. Insaccato dolce fatto con sangue di maiale. Di etimo incerto. Voce endemica (Moscarda Mirković 2015: 128-129), der. forse da *mūla 'stomaco' di orig. or. (REW 5724). Il FEW propende per un traslato dal nome dell'animale 'mula', la femmina del mulo (cfr. FEW 6/3, 198). Per Crevatin è sicuramente parola antica, non medievale e secondo lo studioso la sua enorme diffusione può essere spiegata solo pensando che facesse parte del sermo castrensis, cioè che fosse usata dai soldati perché quel cibo (carne conservata) era presumibilmente comune nel loro rancio. Ed è più che probabile che in quel tipo di lingua ci fossero espressioni figurate e metaforiche. Il VG attesta, oltre alla voce mula, le varianti, mulisa, muliza e mulza (VG 659-661) che derivano dalla voce dial. slov. mulca 'sanguinaccio con ripieno di sangue suino, farina di mais, uvetta, zucchero e spezie'.

chi guarda cartelo no magna vitelo - l'espressione è solitamente interpretata come una constatazione dell'alto costo della carne di vitello, per cui se non si vuol spendere non si può pretendere di mangiare bene. L'interpretazione è errata: il modo di dire era corrente nella Venezia del 1750 circa, Chi varda el cartello no magna el vedello e si riferiva al prezzo calmierato introdotto per legge a Venezia. Il calmiere fissava i prezzi di vari generi, prezzi che erano obbligatori per i venditori: costoro però preferivano sostenere di non avere il genere richiesto piuttosto che venderlo al prezzo stabilito dalle autorità, per cui chi 'guardava il cartello', ossia l'acquirente che voleva pagare il prezzo calmierato e non sottobanco, non avrebbe 'mangiato vitello', perché gli sarebbe stato detto che non ce n'era. (cfr. Zorzi Muazzo 2008). 


\section{Fasi successive e conclusione}

Alla conclusione delle interviste in tutte le località previste come punti d'indagine, seguirà la parte dedicata alle trascrizioni ortografiche dei singoli lemmi del campo semantico della 'casa' e in un secondo momento si procederà alle trascrizioni fonetica e fonologica, con l'aggiunta delle annotazioni e delle indicazioni linguistiche, sociolinguistiche o altre pertinenti alla lettura delle verbalizzazioni.

Gli studiosi che stanno partecipando al progetto stanno riflettendo sull'organizzazione di una banca dati. Si prevede la realizzazione di un Archivio della memoria linguistica e culturale istriana digitalizzato, progettato in modo da avere al suo interno una quantità di dati linguistici e metalinguistici differenti, organizzati in modo da permettere processi di comparazione. La consultazione prevedrà l'accesso alla singola intervista con tutti i diversi sistemi di trascrizione e di analisi, che consentirà di combinare l'analisi quantitativa e l'analisi qualitativa.

Le interviste sinora condotte hanno sin da subito fatto emergere sul territorio istriano una realtà linguistica dialettale plurima e sfaccettata. La volontà degli studiosi che partecipano alle ricerche del progetto AMLCI è quella di conservare tale ricchezza inter e intra-individuale in tutta la sua potenzialità conoscitiva.

\section{Bibliografia}

Balbi, Maria; Moscarda Budić, Maria (2003). Vocabolario del dialetto di Gallesano d'Istria. Collana degli Atti, 20. Trieste-Rovinj/Rovigno: Centro di ricerche storiche.

Bartoli, Matteo Giulio et al. (diretto da) (1995 ssg.). Atlante linguistico italiano. Centro per 1'ALI. Torino: Università degli Studi di Torino.

Berruto, Gaetano (2010). Fondamenti di sociolinguistica, Bari: Laterza.

Buršić-Giudici, Barbara (2009). La vita rustica di Sissano rispecchiata nel suo dialetto. Pula/Pola: Pietas Iulia, Rijeka/Fiume: Edit.

Buršić-Giudici, Barbara (2011). La terminologia vitivinicola da Pellis ad oggi. In «Tabula» 9, Pula/Pola: Sveučilište Jurja Dobrile u Puli, Odjel za humanističke znanosti. Buršić-Giudici, Barbara / Giuseppe Orbanich (2009). Dizionario del dialetto di Pola. Collana degli Atti, 31. Trieste-Rovinj/Rovigno: Centro di ricerche storiche.

Cergna, Sandro (2015). Vocabolario del dialetto di Valle d'Istria, Collana degli Atti, 41. Rovinj/Rovigno: Centro di ricerche storiche.

Cernecca, Domenico (1967). Analisi fonematica del dialetto di Valle d'Istria. In: Studia Romanica et Anglica Zagrabiensia 23. Zagreb: Filozofski fakultet. 137-160.

Cernecca, Domenico (1976). Formazione delle parole nell'istrioto di Valle d'Istria. In: Studia Romanica et Anglica Zagrabiensia. Zagreb: Filozofski fakultet. 41-42.

Cernecca, Domenico (1986). Dizionario del dialetto di Valle d'Istria. Collana degli Atti, 8. Trieste-Rovinj/Rovigno: Centro di ricerche storiche.

Cortelazzo, Manlio (1972). Tracce dell'antico dialetto veneto di Pirano. In: Linguistica 12, Ljubljana: Filozofska fakulteta v Ljubljani. 31-40. 
Cossutta, Rada / Crevatin Franco (2006). Slovenski dialektološki leksikalni atlas slovenske Istre, II, Koper: Založba Annales.

Crevatin, Franco (1982). I dialetti veneti dell'Istria. In: Guida ai dialetti veneti IV. 39-50.

Crevatin, Franco (1999). Note etimologiche italoromanze. In: Incontri Linguistici 22. 177-179.

D’Agostino, Mari / Paternostro, Giuseppe [a cura di] (2006). Costruendo i dati: metodi di raccolta, revisione e organizzazione della banca nella selezione sociovarazionale. Palermo: Materiali e ricerche dell' ALS, 17.

Dalla Zonca, Giovanni Andrea (1978). Vocabolario dignanese-italiano. Collana degli Atti, 2. Trieste-Rovinj/Rovigno: Centro di ricerche storiche.

Deanović, Mirko (1955). Istroromanske studije. Rad JAZU 303. Zagreb: JAZU.

Deanović, Mirko (1960). Sull'istrioto. In: Atti del VII Congresso internazionale di studi romanzi II. 505-513.

Deanović, Mirko (1962). Tracce dell'istrioto nell'antica toponomastica dell'Istria. In: Atti e memorie della sezione toponomastica, Firenze: I - VII Congresso internazionale di scienze onomastiche.

Dussich, Marino (2019). Dizionario italiano-buiese. Collana degli Atti, 46. Rovinj/ Rovigno: Centro di ricerche storiche.

FEW = Von Wartburg, Walther (1922-1989). Französisches etymologisches Wörterbuch: eine Darstellung des galloromanischen Sprachschatzes. Bonn-Leipzig: TübingenBasel <https://apps.atilf.fr/lecteurFEW/index.php/page/view (25/03/2020).

Filipi, Goran (1989b). Situazione linguistica Istro-quarnerina. In: Ricerche sociali 1. Rovinj/Rovigno: Centro di ricerche storiche. 73-82.

Filipi, Goran (1993). Istriotski jezikovni otoki v Istri. In: Annales - Anali za istrske in mediteranske študije 3. Koper: Series Historia et Sociologia. 275-284.

Filipi, Goran (1994). Le parlate istriote. In: La Battana 111. Rijeka/Fiume: Edit. 83-88.

Filipi, Goran (1996). Dialettologia istriana. In: Scuola Nostra 26. Rijeka/Fiume: Edit. 113-121.

Filipi, Goran / Buršić-Giudici, Barbara (1998). Istriotski Lingvistički Atlas - Atlante Linguistico Istrioto. Pula: Znanstvena udruga Mediteran.

Filipi, Goran / Buršić-Giudici, Barbara (2012). Istromletački lingvistički atlas - Atlante Linguistico Istroveneto. Zagreb: Dominović.

Glavinić, Vera (2000). Vocabolario del dialetto istroveneto di Pola. Pula/Pola: Filozofski fakultet u Puli.

Gobo, Giampietro ( ${ }^{32002) . ~ L e ~ r i s p o s t e ~ e ~ i l ~ l o r o ~ c o n t e s t o . ~ P r o c e s s i ~ c o m u n i c a t i v i ~ e ~ c o g n i t i v i ~}$ nelle interviste standardizzate, Milano: Franco Angeli.

Manzini, Giulio / Rocchi, Luciano (1995). Dizionario storico fraseologico etimologico del dialetto di Capodistria. Collana degli Atti, 12. Trieste-Rovinj/Rovigno: Centro di ricerche storiche.

Martinet, André (2008). Introduzione a Uriel Weinreich, in: Lingue in contatto, Orioles, Vincenzo [a cura di] Torino: Utet Università, pp. CVII-CVIII.

Matranga, Vito (2002). Come si fa un'indagine dialettale sul campo, in: I dialetti italiani. Storia, struttura, uso, AA. VV., [a cura di] Torino: Utet. 
Moscarda Mirković, Eliana / Moscarda, Luana (2015). Sulle orme della tradizione culinaria gallesanese. Aspetti culturali e storico linguistici. Trieste-Rijeka (Fiume): Università Popolare di Trieste, Unione Italiana-Fiume.

Pellegrini, Gian Battista (1972 ssg.). Atlante Storico Linguistico Etnografico Friulano. Udine: Istituto di Glottologia e Fonetica dell'Università di Padova, Istituto di Filologia romanza della Facoltà di Lingue e Letterature Straniere di Trieste. Pellizzer, Antonio / Pellizzer, Giovanni (1992). Vocabolario del dialetto di Rovigno d'Istria, Collana degli Atti, 10. Trieste-Rovinj/Rovigno: Centro di ricerche storiche.

Prati, Angelico (1951). Vocabolario etimologico italiano. Torino: Multigrafica editrice. REW = Meyer-Lübke, Wilhelm (72009). Romanisches etymologisches Wörterbuch. Heidelberg: Carl Winter Universitätsverlag.

Tekavčić, Pavao (1976). Per un atlante linguistico istriano (Con speciale riguardo ai dialetti istroromanzi). In: Studia Romanica et Anglica Zagrabiensia. Zagreb: Filozofski fakultet. 41-42.

Todorović, Suzana (2019). L'istroveneto nell'ambito degli altri idiomi delle località slovene costiere. Koper: Libris.

Todorović, Suzana (2020). Atlante linguistico istroveneto dell'Istria nordoccidentale. Koper: Libris.

VG = Rosamani, Enrico (21999), Vocabolario giuliano dei dialetti parlati nella Venezia Giulia, in Istria, in Dalmazia, a Grado e nel Monfalconese. Trieste: Lint.

Zorzi Muazzo, Francesco (2008). Raccolta de' proverbii, detti, sentenze, parole e frasi veneziane, arricchita d'alcuni esempii ed istorielle, Crevatin, Franco [a cura di] Costabissara: Colla Editore.

\section{Dijalekti u doticaju u Istarskoj županiji. Metode istraživanja u sklopu Arhiva jezične i kulturalne baštine Istre}

Globalizacija i promjena ekonomskih i socijalnih uvjeta ugrožavaju bogatu nematerijalnu baštinu koja je zapravo okosnica svake povijesne studije. Upravo se zbog toga u sklopu projekta Arhiv jezične i kulturne baštine Istre (nastao kroz suradnju Sveučilišta Jurja Dobrila u Puli i Sveučilišta u Trstu) provodi istraživanje čiji je cilj postupno uspostavljanje jezičnog arhiva iz različitih semantičkih polja, dostupnog svim znanstvenicima i zainteresiranima, koji će dokumentirati jezični razvoj kroz povijest hrvatskog, talijanskog i slovenskog naroda na istarskom poluotoku. Ovaj rad predstavlja ukratko faze istraživanja koje su do sada provedene u okviru projekta, u vidu prostora za proučavanje i očuvanje baštine te promoviranje razvoja kulture i jezika (posebice narječja) na istarskom području.

Ključne riječi: arhiv, Istra, manjinski jezici, jezici u doticaju, narječja 